\title{
Bilateral sleeve lobectomy for synchronized bilateral central carcinoid tumor
}

\author{
Gokhan Yuncu, MD, ${ }^{\text {a }}$ Serpil Sevinc, MD, ${ }^{a}$ Berna Ozturk, MD, ${ }^{\mathrm{b}}$ Goksel Kiter, MD, ${ }^{\mathrm{c}}$ Ferda Bir, MD, ${ }^{\mathrm{d}}$ and \\ Nevzat Karabulut, MD, ${ }^{e}$ Denizli, Turkey
}

$\mathrm{W}$

e report an unusual case of synchronized bilateral central carcinoid tumors removed by means of bilateral sleeve lobectomy. Because they have a relatively benign course, lung-sparing procedures are advisable for centrally located carcinoid tumors when possible.

\section{Clinical Summary}

A 34-year-old man complained of cough, dyspnea, and fever for 8 months and was investigated for newly occurred hemoptysis.

Chest radiography showed left hilar enlargement and heterogeneous infiltration in the left upper zone. An endobronchial mass obliterating the left upper lobe (LUL) bronchus accompanied with LUL atelectasis and a $7-\mathrm{mm}$ mass in the right intermediate bronchus was seen on computed tomography (Figures 1 and 2).

Bronchoscopy revealed a vegetative mass obliterating the LUL bronchus and protruding to the left main bronchus and a polypoid lobular mass at the medial part of the right intermediate bronchus partially occluding the right middle lobe bronchus. The biopsy specimens of both the right- and left-sided lesions have been reported as typical carcinoid tumor (TCT).

First, single-lung ventilation with a double-lumen tube and left-sided posterolateral thoracotomy were performed. A $3 \times$ $2-\mathrm{cm}$ solid mass was palpated at the distal left main bronchus extending to the proximal left upper and lower lobe bronchi during the exploration. A left upper-sleeve lobectomy and radical lymph node dissection (RLND) were performed.

The second operation was performed after 10 weeks and after a fiberoptic bronchoscopy that showed an intact previous anastomosis line and an unchanged right-sided tumor. That time, singlelung ventilation was not preferred, and the patient was intubated through a single-lumen endotracheal tube. A sleeve resection of the right intermediate bronchus and middle lobe and RLND were performed.

\footnotetext{
From the Medical Faculty, Thoracic Surgery Department, ${ }^{\text {a }}$ Chest Department, ${ }^{\mathrm{c}}$ Pathology Department, ${ }^{\mathrm{d}}$ and Radiology Department, ${ }^{\mathrm{e}}$ Pamukkale University, and the Chest Clinic, Denizli Government Hospital, ${ }^{\mathrm{b}}$ Denizli, Turkey.

Received for publication Dec 9, 2006; revisions received Jan 5, 2007; accepted for publication Jan 23, 2007.

Address for reprints: Goksel Kiter, MD, Pamukkale University, Medical Faculty, Chest Department, Kinikli 20070 Denizli, Turkey (E-mail: gokselkiter@yahoo.com).

J Thorac Cardiovasc Surg 2007;133:1660-1

$0022-5223 / \$ 32.00$

Copyright $\odot 2007$ by The American Association for Thoracic Surgery doi:10.1016/j.jtcvs.2007.01.033
}

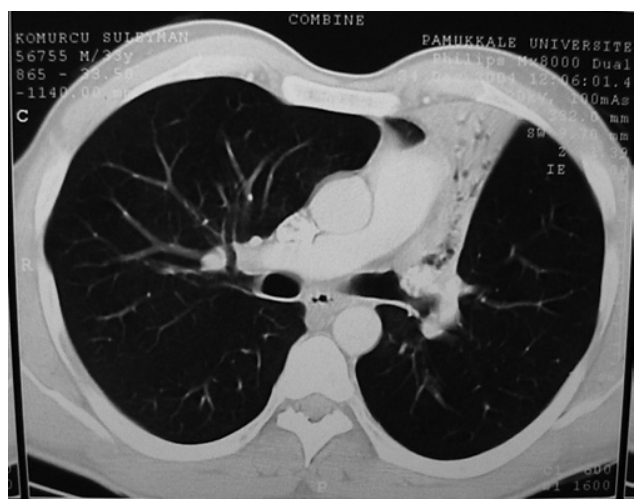

Figure 1. Chest computed tomographic scan demonstrates an occluded left upper lobe bronchus causing distal atelectasis.

Both histologic and immunohistochemical examinations of the operation materials confirmed TCT. The resected right middle lobe and LUL revealed bronchiectasia. No malignant cell was found in any lymph node or surgical margins. The patient was alive and disease free at his latest control visit 20 months after the last operation.

\section{Discussion}

In the literature, only one case of bilateral synchronized carcinoid tumors has been reported. ${ }^{1}$ Because those bilateral tumors were peripheral lung nodules, formal right upper lobectomy and left lower lobectomy as staged procedures had been satisfactory. A case of synchronized bilateral carcinoids that included lingulectomy for atypical carcinoid and right upper lobectomy for typical carcinoid has also been reported. ${ }^{2}$

TCTs are potentially curable, even when they occur synchronously in both lungs. However, careful and detailed preoperative planning plays a crucial role for aggressive management. Anatomic resection with RLND should be considered appropriate treatment. El Jamal and colleagues ${ }^{3}$ have concluded that the policy of conservative resection is feasible and safe when the true histology is known and the distal lung parenchyma is functional.

Although low-grade tumors seem to be a risk in patients with carcinoid tumors, having them at bilateral and central locations changes the treatment decision making. Because the standard resections of left pneumonectomy and right inferior bilobectomy are impossible and bilateral sleeve resections are mandatory for curative treatment, the preoperative evaluation of our patient established the need to analyze some specific variables: the risk for necessity of consecutive resections, the priority of the side for the 


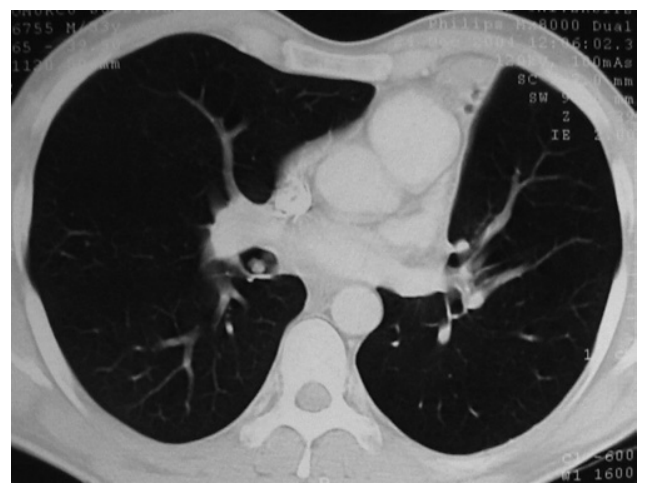

Figure 2. Computed tomographic scan at the caudal level reveals a lobulated endobronchial mass within the intermediate bronchus.

first operation, the ideal time interval between the operations, and the use of anesthesia.

If the right-sided resection had been performed first, doublelumen intubation and independent lung ventilation would have been feasible for the subsequent left-sided operation. But the left side had to be chosen for the first sleeve resection because a more advanced lesion caused hemoptysis and a postobstructive pneumonia was present in the LUL.

Bilateral sleeve resections have been reported previously for neither carcinoids nor synchronized lung carcinomas. Bilateral sleeve resections with a 5-year interval have been reported in a patient with squamous lung cancer as a very rare condition. ${ }^{4}$ Therefore, our case is not only the first case of synchronized bilateral central carcinoids but also the first case of bilateral sleeve resections with a 10 -week interval.

It was important to discuss the case with the anesthesiologist while planning the most appropriate procedure. Because it was just 10 weeks from the first resection, single-lung ventilation was not preferred. Thus, the patient was prevented from incurring the possible risk of damage to the suture line and insufficient ventilation with one remaining lobe of the left lung. Additionally, the right upper lobe ventilation during the anastomosis could be achieved favorably.

Ideal timing for staged operations was not suggested previously. An interval of 2 or 3 months would be tolerated well, as seen in our case.

The best surgical treatment for central carcinoids is a lungsaving procedure such as a staged bilateral sleeve resection that can be performed safely, even within a short interval.

\section{References}

1. Beshay M, Roth T, Stein R, Schmid RA. Synchronous bilateral typical pulmonary carcinoid tumors. Eur J Cardiothorac Surg. 2003;23:251-3.

2. Spaggiari L, Veronesi G, Gasparri R, Pelosi G. Synchronous bilateral lung carcinoid tumors: a rare entity? Eur J Cardiothorac Surg. 2003; 24:334-7.

3. El Jamal M, Nicholson AG, Goldstraw P. The feasibility of conservative resection for carcinoid tumours: is pneumonectomy ever necessary for uncomplicated cases? Eur J Cardiothorac Surg. 2000;18:301-6.

4. Watanabe Y, Kobayashi H, Murakami S, Sawa S, Shinagawa M, Iwa T. Bilateral sleeve lobectomy for metachronous multiple primary lung cancer. Jpn J Surg. 1986;16:56-61. 